\title{
Carbon Nanotube-Textured Sand for Controlling Bioavailability of Contaminated Sediments
}

\author{
Xingmao Ma ${ }^{1}(\varangle)$, Deepti Anand ${ }^{1}$, Xianfeng Zhang ${ }^{2}$, Mesfin Tsige $^{2}(\varangle)$, and Saikat Talapatra ${ }^{2}(\varangle)$ \\ ${ }^{1}$ Department of Civil and Environmental Engineering, Southern Illinois University Carbondale, IL 62901, USA \\ ${ }^{2}$ Department of Physics, Southern Illinois University Carbondale, IL 62901, USA \\ Received: 30 March 2010 / Revised: 9 April 2010 / Accepted: 11 April 2010 \\ (C) The Author(s) 2010. This article is published with open access at Springerlink.com
}

\begin{abstract}
Sand particles textured with multi-walled carbon nanotubes (MWCNTs) can efficiently control the mobility and bioavailability of contaminants found in aquatic sediments. Adsorption measurements for a wide variety of aquatic contaminants (chlorinated hydrocarbons) on MWCNT-textured sand showed orders of magnitude increase in their sorption coefficients compared to traditional materials (sand) when used for physically separating contaminated sediments from overlying water. Molecular dynamics simulations performed on model experimental systems emphasize that the hydrophobic interactions of the MWCNT surfaces play a crucial role in driving the water molecules away, promoting such enhanced contaminant uptake. The MWCNT-textured sand significantly reduced the migration of contaminants from sediments to overlying water and possesses suitable parameters needed for contaminant sequestration and sediment remediation technologies. The single step and scalable procedure described here for synthesizing robust MWCNT-textured sand surfaces will provide important improvements in the field of remediation/aquatic environment restoration technologies.
\end{abstract}

\section{KEYWORDS}

Multi-walled carbon nanotube (MWCNT)-textured sand, in situ capping, capping amendments, chlorinated compounds

\section{Introduction}

Significant amounts of underwater sediments are sufficiently contaminated to pose potential risks to aquatic creatures and human beings according to a United Sates Environmental Protection Agency (EPA) survey [1]. Contaminated sediments often function as a long term pollution source for surface water because sediments frequently release adsorbed contaminants back to the pore water. Some of these contaminants, which include a wide array of organic and inorganic contaminants such as trichloroethylene (TCE), polycyclic aromatic hydrocarbons (PAHs), polychlorinated biphenyls (PCBs), mercury ( $\mathrm{Hg})$, arsenic (As), etc., are hazardous to aquatic life as well as to human beings. Remediation of contaminated sediments is extremely challenging and often requires complex sediment remediation technologies such as dredging, in situ or ex situ capping and monitored natural attenuation (MNA) [2]. Dredging is an effective way to reduce contaminant masses, but its long term effectiveness in reducing risks is uncertain [3]. Furthermore, in

Address correspondence to Xingmao Ma,ma@engr.siu.edu; Saikat Talapatra, stalapatra@physics.siu.edu;

Mesfin Tsige, mtsige@physics.siu.edu 
the short term, many adverse effects such as elevated contaminant levels in caged fish adjacent to dredging activities have been reported due to the residue plumes left by dredging [2,4]. MNA (a process which controls or removes pollutants through natural processes, such as consumption of contaminants by microorganisms that are present in the contaminated soil) is a cost-effective technology, but the time span it requires is too long to be practical in many contaminated sites. Due to these reasons, processes such as in situ capping (ISC) are desirable and sometimes are necessary to physically separate contaminants in sediments from the overlying water in order to reduce their bioavailability to benthic organisms in water and to maintain the economic value of the water bodies. The most commonly used capping materials are sand and clean sediments. However, efficacies of these traditional ISC materials are severely limited when it comes to capping soft or highly erosive sediments [5]. Materials such as organoclays and zeolites have been evaluated by different researchers but none of these were found to be adequate to capture contaminants from sediments $[5,6]$. Hence, there is a critical need to develop better active or reactive cappings with the potential to significantly reduce the contaminant mass and/or the bioavailability of contaminants without affecting the soft sediments. In this article we report the synthesis of a novel reactive capping material, namely multi-walled carbon nanotube (MWCNT)textured sand particles and show that the MWCNTtextured sand can act as a highly effective ISC material. Carbon nanotubes (CNTs) possess very attractive physical properties (due to their nanometer dimensions) for a wide variety of applications $[7,8]$. One such property that makes them excellent materials for various adsorption-based applications is the availability of high specific surface area $[9,10]$. Although CNTs and their composites have been widely studied for various applications [11], their superior surface properties have not been fully explored for environmental cleaning applications, in spite of some reports of the use of CNTs for contamination removal showing that they can be better adsorbents than activated carbons [12]. For example, Long et al. [13] reported that CNTs have significantly higher dioxin removal efficiency than that of activated carbon. Similarly, heavy metal removal from water using CNTs also indicated their superiority as contaminant removal agents $[14,15]$. These results point towards the possibility of using CNTs for other applications such as to efficiently control the mobility and bioavailability of contaminants found in aquatic sediments, or in other words as a component of an in situ capping material. In this study we have shown that MWCNTtextured sand particles can effectively remove a wide variety of chlorinated aliphatic contaminants. Investigations have shown that the migration of the contaminants back to water is also sufficiently reduced once adsorbed on to the MWCNT-textured sand. Molecular dynamics (MD) simulations performed on a model experimental system showed that the surface chemistry of MWCNTs plays a crucial role in separating and controlling the migration of contaminants in water. The MWCNT-textured sand possesses the required geotechnical properties needed for ISC materials and the attachment of the MWCNTs to the sand particles is strong enough to survive vigorous agitation, indicating the mechanical stability of the MWCNT-sand interface.

\section{Results and discussion}

The nano-texturing of the sand surface with MWCNTs was achieved by using a recently reported [16] airassisted chemical vapor deposition process (details of the synthesis are provided in the Materials and methods section). Representative growth results of MWCNT-textured sand synthesized under various conditions are shown in Fig. 1. Figure 1(a) shows optical images of as-received sand particles and MWCNT-coated sand particles. A scanning electron microscopy (SEM) image of a few sand particles is shown in Fig. 1(b). Figures 1(c) and 1(d) show the MWCNTs grown on sand particles under two different growth conditions. In Fig. 1(c), the MWCNT growth was obtained without using any air and in Fig. 1(d), 5 standard cubic centimeters per minute $(\mathrm{sccm})$ of air was introduced during the growth process (all other growth conditions were same for both the samples). Our recent reports have indicated that introduction of air during the growth process of MWCNTs substantially helps the MWCNT growth rate. This is 

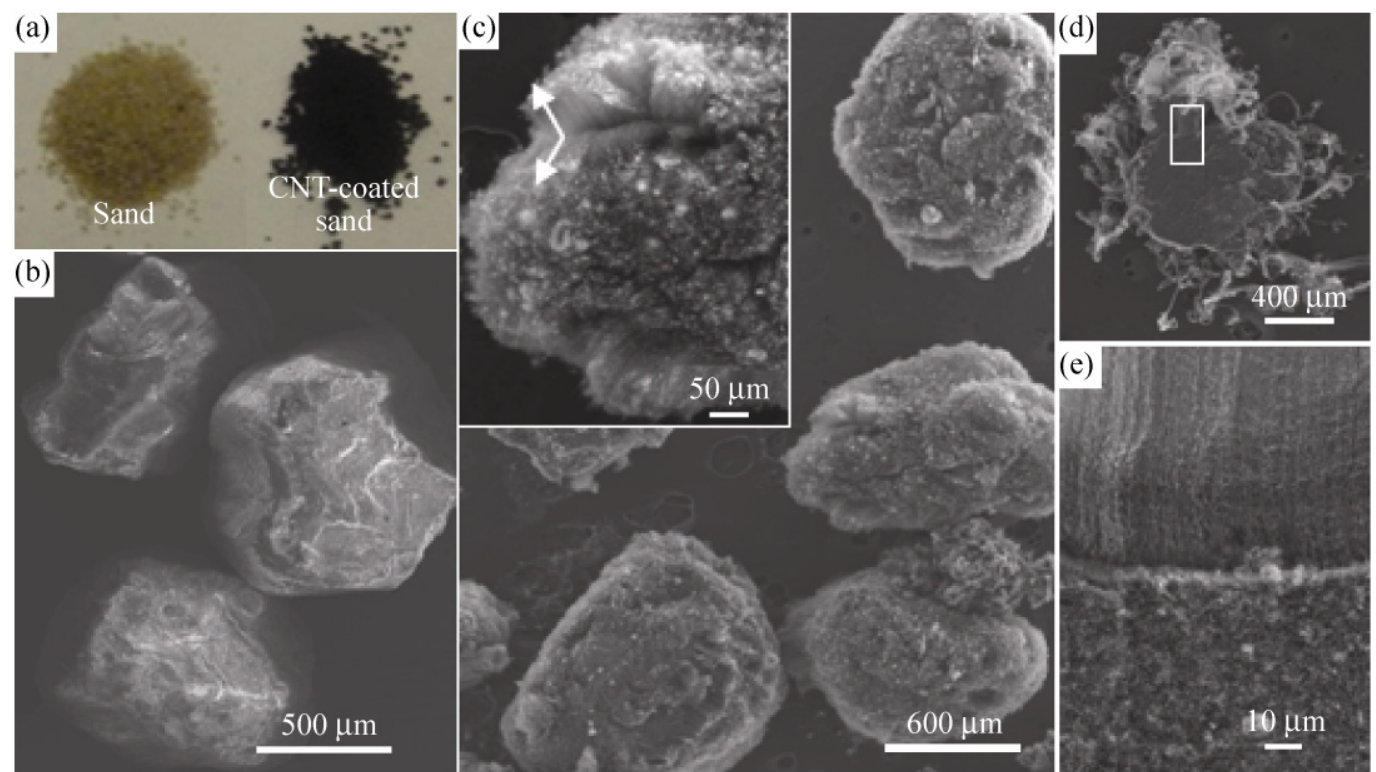

Figure 1 MWCNT-textured sand: (a) optical image of a small pile of as-received sand and MWCNT-coated sand; (b) scanning electron microscopy of sand particles showing bright regions due to charging effects under the electron beam; (c) MWCNT-coated sand (The inset shows a higher magnification image of one of the sand particles. The MWCNT growth direction is shown by the arrow); (d) a sand particle coated with long MWCNT bundles grown with an air flow rate of $5 \mathrm{sccm}$; (e) a higher magnification of the region shown with a white square in (d) showing dense architectures of MWCNTs on sand surfaces

also evident here from the lengths of the MWCNT bundles obtained, shown in Fig. 1(d). Figure 1(e) shows a higher magnification SEM image of a portion of the sample shown in Fig. 1(d).

The sorption capacities of nano-textured sand were assessed with one of the most prevalent sediment contaminants, TCE. TCE adsorption coefficients were measured (see Materials and methods section for the details of the measurements) to determine the optimal nano-textured sand product for contamination removal. The results of the TCE adsorption measurements on MWCNT-textured sand produced using different air flow rates and growth temperatures are shown in Fig. 2(a) (other MWCNT growth parameters, such as growth time, carrier gas flow rates, etc., were kept constant for the data set shown in this figure). Based on our results, we conclude that the optimal quality of nano-textured sand for TCE removal was achieved using the following MWCNT growth conditions: growth temperature $\mathrm{T}, \sim 790{ }^{\circ} \mathrm{C}$; air flow, $\sim 5.0 \mathrm{sccm}$; $\mathrm{Ar} / \mathrm{H}_{2}$ flow, $\sim 400 \mathrm{sccm}$; and growth time, $\sim 30 \mathrm{~min}$. Large amounts of nano-textured sand were grown under these conditions and adsorption measurements were performed to determine the sorption coefficients $\left(K_{d}\right)$ of a series of homologous chlorinated ethenes and chlorinated ethanes on the material. We also measured the $K_{d}$ values for the same contaminants on bare sand and compared them with the values obtained on MWCNT-textured sand at the same concentration. The results are shown in Fig. 2(b). The sorption coefficients of the tested chlorinated aliphatics on MWCNT-textured sand were at least an order of magnitude higher than their sorption coefficients on bare sand. In some cases-for example, 1,1,1trichloroethane (where the sorption coefficient of $3.71 \mathrm{~L} / \mathrm{kg}$ for bare sand compared with $148 \mathrm{~L} / \mathrm{kg}$ for MWCNT-textured sand) - there was an increase of more than one order of magnitude in sorption coefficient.

In order to have a molecular-level insight into the fundamental interactions between contaminants and the surfaces, atomistic MD simulations were carried out on systems containing water and TCE mixtures on MWCNTs as well as on silica surfaces. Computer simulations, especially molecular dynamics simulations, have played a major role in developing our understanding of the molecular level structure of interfaces (liquid/vapor, liquid/liquid, and liquid/solid) 
and other fine details of the interface which may not be easily obtained from experiments. The systems simulated here consist of a water/TCE mixture over a silica substrate and over an MWCNT surface as shown in Fig. 3. The concentration of TCE in the mixture was varied between $2 \%$ and $40 \%$, while keeping the number of water molecules (2000) in the mixture constant. The amorphous silica in our simulation is hydroxylated $\left(4.6 \mathrm{OH} / \mathrm{nm}^{2}\right)$, since in reality the formation of silanol groups is known to occur rapidly

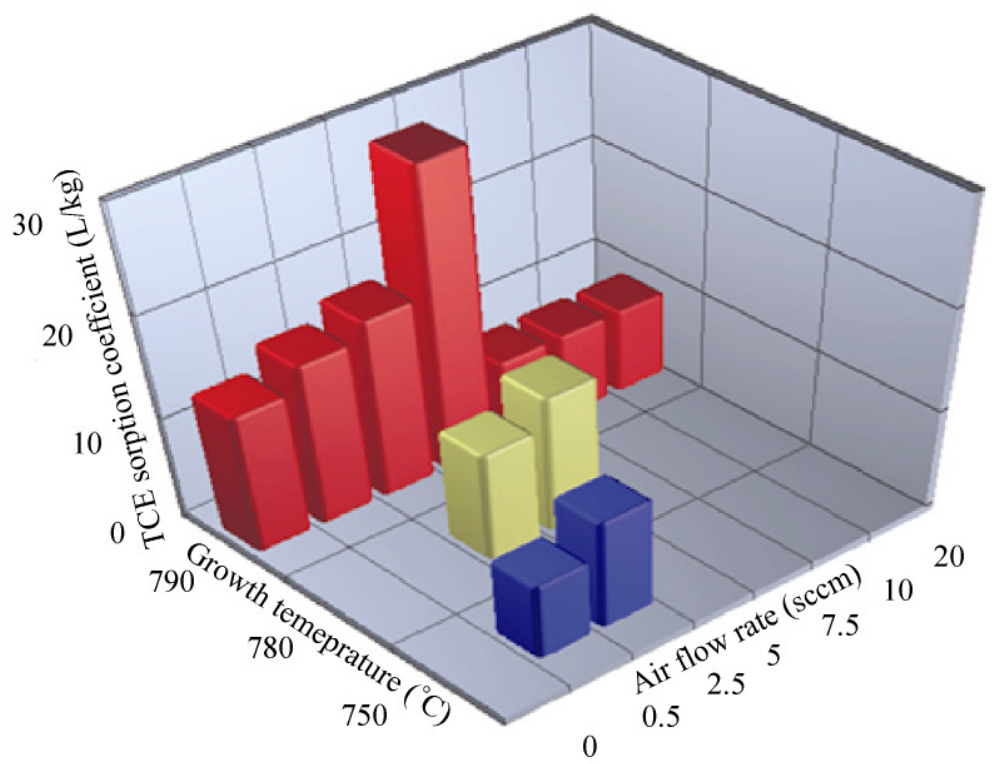

(a)

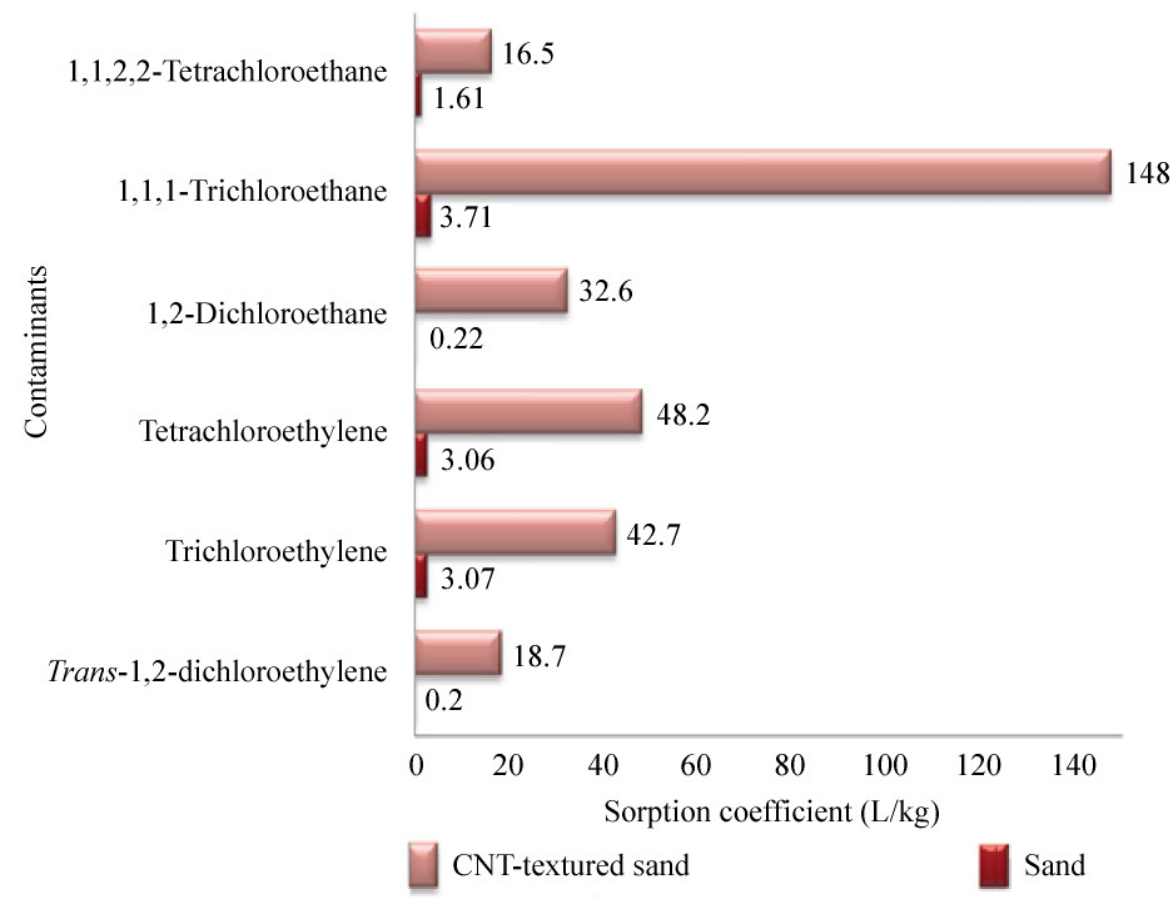

(b)

Figure 2 Adsorption coefficient of TCE on MWCNT-coated sand: (a) the graph shows the TCE adsorption coefficient of nano-textured sand performed on a variety of samples grown under different synthesis conditions to determine the optimal surface property of the MWCNTs needed for this specific application; (b) comparison of the adsorption coefficients obtained for six different chlorinated aliphatics on sand and MWCNT-textured sand manufactured under the optimal conditions shown on (a) 
when a freshly formed silica surface is exposed to atmospheric moisture.

Results from MD simulation studies are presented in Fig. 3. The left column of Fig. 3 shows snapshots at the end of $4 \mathrm{~ns}$ molecular dynamics simulations of the different water/TCE mixtures adsorbed on a hydroxylated amorphous silica surface. The simulation studies of the adsorption of the water/TCE mixtures on MWCNTs are shown in the right column. In the figure, the top, middle, and bottom rows correspond

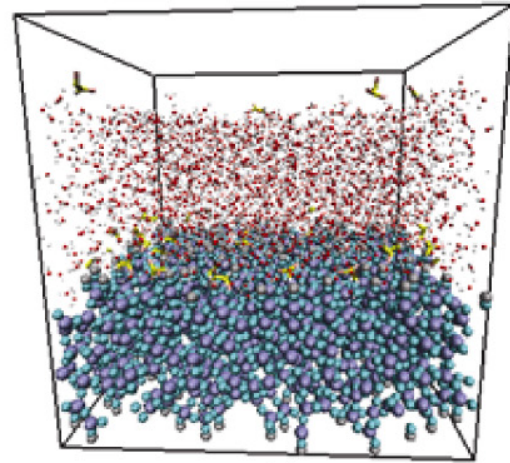

(a)

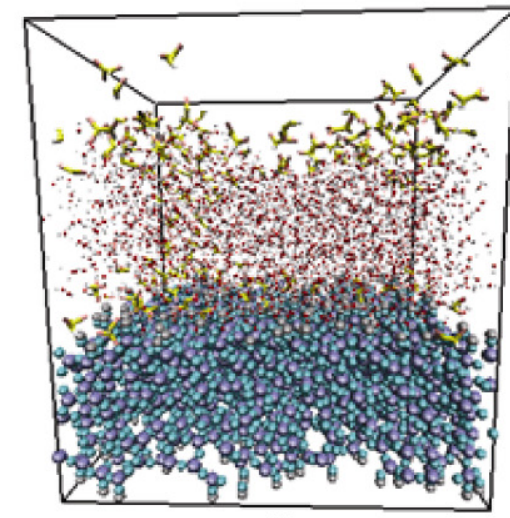

(b)

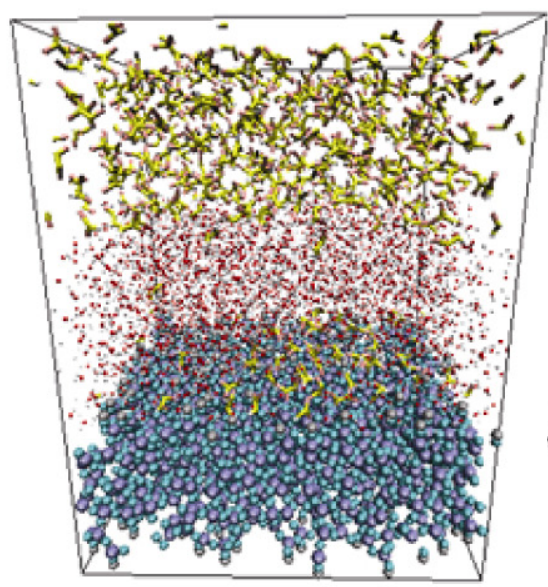

(c)

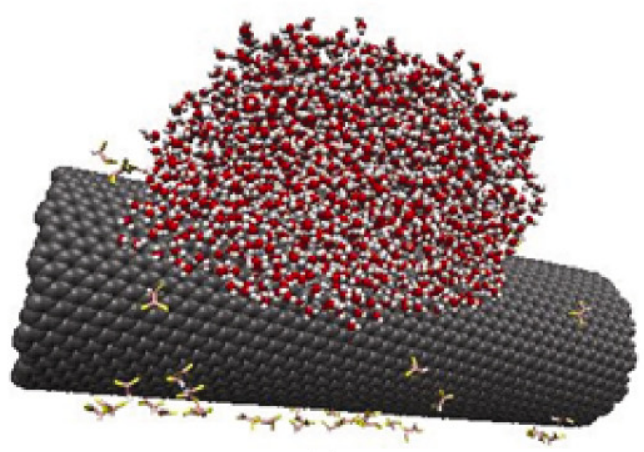

(d)

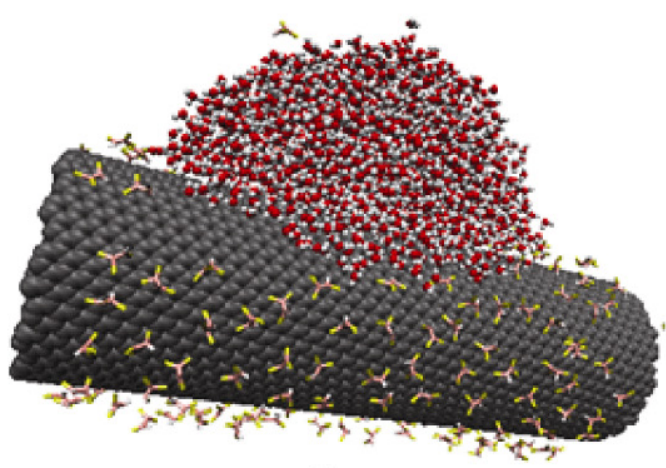

(e)

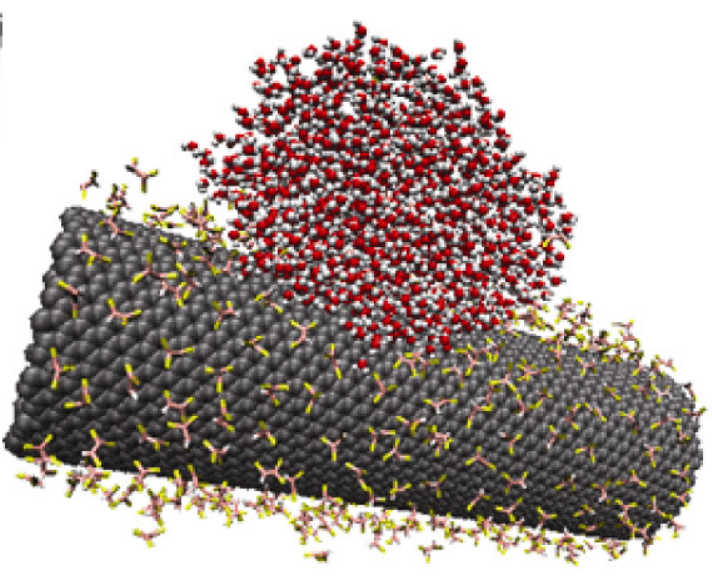

(f)

Figure 3 Simulation snapshots of adsorption of TCE-water mixtures on silica surfaces and MWCNTs. The left column of the figure shows snapshots at the end of 4 ns molecular dynamics simulations of the different water/TCE mixtures adsorbed on a hydroxylated amorphous silica surface (a)-(c) and carbon nanotubes (d)-(f). The concentration of TCE was varied in performing the MD simulations. In the figure, the top $(\mathrm{a}, \mathrm{d})$, middle $(\mathrm{b}, \mathrm{e})$, and bottom $(\mathrm{c}, \mathrm{f})$ rows correspond to $2 \%, 15 \%$, and $40 \%$ concentrations of TCE in the mixture, respectively 
to $2 \%, 15 \%$, and $40 \%$ TCE concentration in the mixture, respectively. There is a general expectation that water should be preferentially adsorbed on a hydrophilic surface from the water/TCE mixtures, and the snapshots clearly show that. If we define the equilibrium selectivity factor of TCE with respect to water by the ratio of the numbers of the two types of molecules adsorbed on the amorphous silica surface, we find a selectivity factor of about 0.1 , independent of the TCE concentration in the mixture. The snapshots show that increasing the TCE concentration in the mixture results in the formation of a TCE cluster on the amorphous silica surface, without significantly changing the number of TCE molecules adsorbed on the surface. Figures 3(b) and 3(c) show that excess TCE molecules move to the air interface since TCE has a much lower surface tension than water.

Increasing the $\mathrm{OH}$ concentration on the silica surface (not shown here) was found to decrease the equilibrium selectivity factor of TCE. For example, on a highly hydroxylated silica surface, $\alpha$-quartz $(9.6$ $\mathrm{OH} / \mathrm{nm}^{2}$ ), we found an equilibrium selectivity factor of 0 . This is clearly due to the huge difference in interaction energies between the two adsorbates and the silanol groups of the silica surfaces.

The simulation studies of the adsorption of water/TCE mixtures on MWCNTs were found to give completely opposite results to those obtained for the same system on silica. The hydrophobic interactions of TCE and the MWCNT surface with water drive the water molecules to form a spherical droplet and as a result it does not wet the surface. The snapshots clearly show that the contact angle of water and the MWCNT surface increases with increasing TCE concentration in the mixture, making the surface superhydrophobic at the highest TCE concentration studied. The TCE molecules adsorb all over the MWCNT surface by continuously displacing the water molecules, and we did not observe any desorption of the TCE molecules in the time scale of our simulation, about $4 \mathrm{~ns}$. The TCE molecules form layers on the MWCNT surface as their concentration in the mixture is increased. These results are solely due to the superior surface properties of the MWCNTs [17, 18]. Thus, dense bundles of MWCNTs covering sand particles provide a huge specific surface area (compared to bare sand) as well as an appropriate attractive surface potential needed to bind the contaminant molecules.

Although the adsorption results obtained for the MWCNT-coated sand indicated that it is a good ISC material, there are several other parameters that are crucial for the successful implementation of ISC materials for sediment remediation. For example, the density of the composites (MWCNT-coated sand) needs to be sufficiently high so that they settle down to the water bed with ease. The calculated densities $\rho_{\text {ns }}$ of the MWCNT-textured sand ranged from 1.1 to $1.58 \mathrm{~g} / \mathrm{mL}$, indicating that they will indeed settle on the top of the sediment once immersed in water. We also measured the porosity of the nano-textured sands, which were in the range $0.278-0.417$, comparable to the porosity of a natural sand bed which is 0.38 . These results indicated that MWCNT-textured sands possess the appropriate geotechnical properties necessary for a capping material and have the ability to settle down on the sediments on which they are placed. Details of the calculation of bulk density $(\rho)$ and porosity $(\varepsilon)$ of the MWCNT-textured sand are mentioned in the Materials and methods section.

Another importation criterion related to effective contamination control is the ability of the sorption agent to prevent the contaminants from reaching the overlaying water layer through pore water migration caused by sediment consolidation after capping. This can be determined by evaluating the retardation factor of the contaminants. The retardation factor $(R)$ is a parameter that indicates how much the contaminant movement is delayed compared with water. Therefore, $R$ is defined as $v_{\mathrm{w}} / v_{\mathrm{c}}$, where $v_{\mathrm{w}}$ is the velocity of water and $v_{\mathrm{c}}$ is the velocity of the contaminant and can be calculated using the equation $R=1+(\rho / \varepsilon) \times K_{\mathrm{d}}$, where $\rho$ is the bulk density and $\varepsilon$ is the porosity. A higher value of $R$ generally represents a better contaminant retardation capability of the adsorbent. Based on the measured bulk density, porosity and sorption coefficients, we calculated the retardation factors for several chlorinated compounds at one-point concentration, which are presented in Table 1 . The calculated values of $R$ ranged from 45 for 1,1,2,2tetrachloroethane to 7883 for 1,3,5-trichlorobenzene, and were significantly higher than those measured on bare sand particles. Since $R$ is dependent upon the 
Table 1 Calculated retardation factors of various contaminants on sand and MWCNT-textured sand

\begin{tabular}{lccc}
\hline & $\begin{array}{c}\text { Molecular } \\
\text { Contaminant }\end{array}$ & $\begin{array}{c}\text { Retardation factor of the } \\
\text { contaminant on }\end{array}$ \\
\cline { 3 - 4 }$\left(\mathrm{cm}^{3} / \mathrm{mol}\right)$ & Sand & $\begin{array}{c}\text { MWCNT- } \\
\text { textured sand }\end{array}$ \\
\hline Trans-1,2-dichloroethylene & 80.2 & 1.78 & 51.8 \\
Trichloroethylene & 98.1 & 12.9 & 117 \\
Tetrachloroethylene & 116 & 12.9 & 132 \\
1,2-Dichloroethane & 87.6 & 1.86 & 89.7 \\
1,1,1-Trichloroethane & 105.5 & 15.4 & 403 \\
1,1,2,2-Tetrachloroethane & 123.4 & 7.26 & 45.9 \\
1,3,5-Trichlorobenzene & 181.5 & 46.5 & 7883 \\
\hline
\end{tabular}

${ }^{\mathrm{a}}$ The molecular size is calculated based on the atomic volume and molecular structure of a molecule ${ }^{[20]}$

sorption coefficient, which varies with the liquid concentration, the adsorption and desorption isotherms of TCE on MWCNT-textured sand were measured, and are shown in Fig. 4. The adsorption isotherm exhibits a typical "L" shape without a strict plateau. We found that, for bare sand, the adsorption capacity is easily saturated, even at low concentrations. There is no desorption hysteresis for TCE from bare sand, but a clear desorption hysteresis was observed for TCE from the MWCNT-textured sand. The strong hysteresis indicates that contaminants will be strongly attached to the MWCNT-textured sand and will not be fully released; therefore, greatly reducing the bioavailability of sediment contaminants to aquatic species. In a natural environment, $R$ can be affected by co-contaminants through competitive adsorption or other environmental factors such as temperature and $\mathrm{pH}$. Details of these variations and their impacts are beyond the scope of the present work and will be addressed separately in the future. However, our present results indicate that the migration of contaminants from sediments to overlying water will be significantly retarded by using MWCNT-textured sand. This is perhaps due to the higher adsorption potentials offered by the curved MWCNT surfaces $[9,19]$ which tend to bind the contaminants more strongly than bare sand. Long retention time of these contaminants in the capping layer is necessary in order for indigenous microorganisms to biodegrade these contaminants. The increased contaminant retardation time is one of the

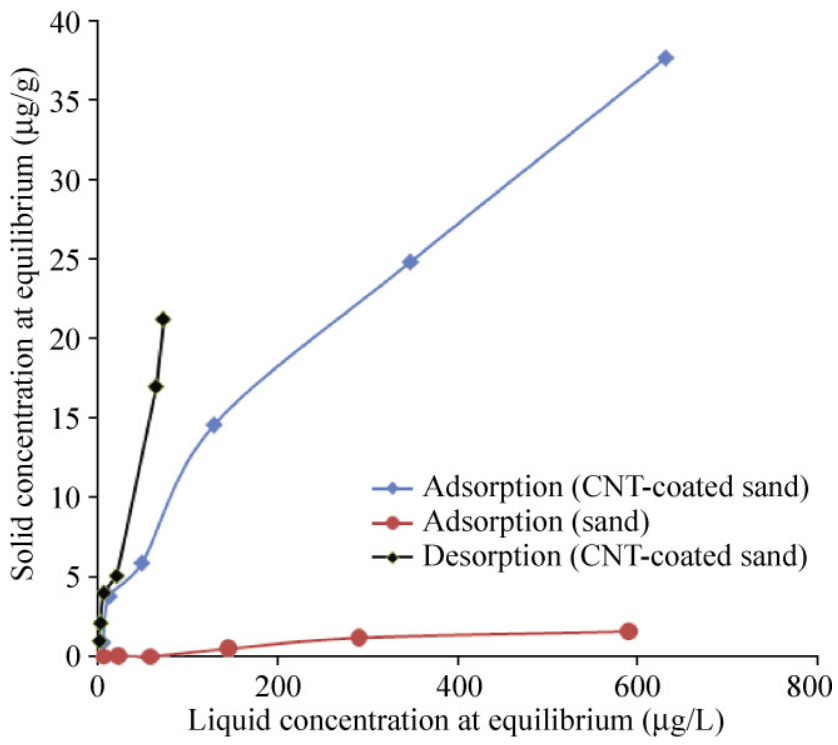

Figure 4 Adsorption-desorption isotherms of TCE on MWCNTcoated sand and bare sand. The isotherms were measured at room temperature $\left(21-23{ }^{\circ} \mathrm{C}\right)$

unique features of the MWCNT-textured sand, and certainly represents a major advantage over traditional capping materials.

The potential uptake of nanomaterials by aquatic plants and benthic organisms has raised much concern recently $[20,21]$. Therefore, the need to assess the stability of the MWCNTs on the sand surfaces becomes important. We evaluated the binding strength of the MWCNTs on the sand surfaces by measuring the turbidity of water after mixing with MWCNT-coated sand and comparing it with the turbidity of pure water as well as that of a water-sand mixture. The turbidity of water containing nano-textured sand ranged from 0.16 to 1.39 nephelometric turbidity units (NTU), with an average of $0.50 \mathrm{NTU}$, whereas the $\mathrm{pH}$ values ranged from 7.14 to 7.55 , with an average of 7.34. The turbidity and $\mathrm{pH}$ of water after mixing and shaking with same amount of bare sand under similar experimental conditions were found to be approximately 1.18 NTU and 7.25, respectively. To establish the importance of direct growth of the MWCNTs on the sand surfaces for contamination uptake, we also performed experiments with physical mixtures of sand and carbon nanotubes. This mixture resulted in similar $\mathrm{pH}$ and turbidity readings; however, it was evident that the MWCNT and sand mixture was very unstable and moderate shaking of this mixture in an aqueous environment 
resulted in the MWCNTs entering the liquid phase. In contrast, we found that the MWCNTs attached to the sand surface were able to survive the agitation provided by a tumbler and remained attached even after the tumbling test. Digital micrographs of the MWCNT and sand mixture in water and MWCNTcoated sand in water after vigorous shaking are shown in Figs. 5(a) and 5(b). SEM images of the MWCNTtextured sand particles after the tumbling test are also shown in Fig. 5.
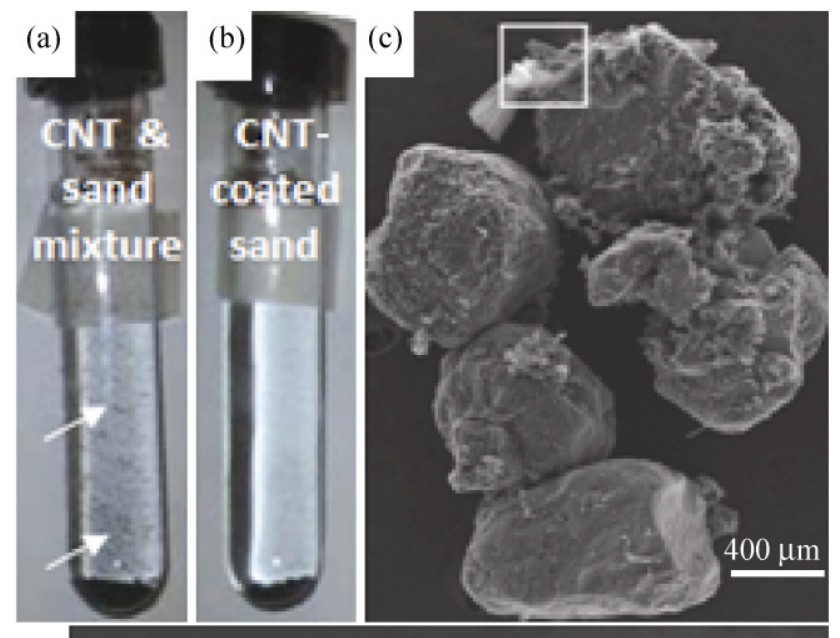

(d)

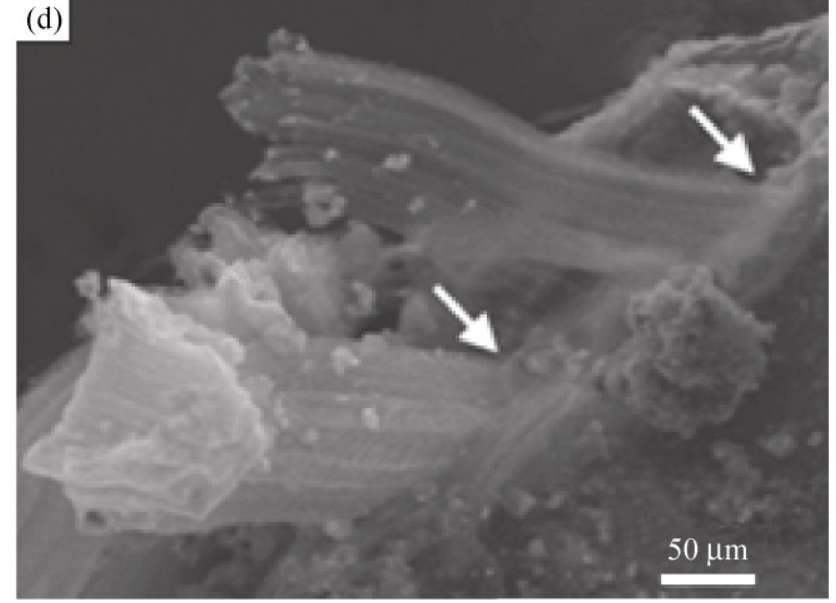

Figure 5 Mechanical stability of MWCNTs grown on sand. Digital micrograph of (a) MWCNT and sand mixture in water and (b) MWCNT-coated sand in water after vigorous shaking. Notice the presence of loose MWCNTs in water (shown using white arrows) in (a). (c) and (d) show SEM images of MWCNT-coated sand particles after performing the tumbling test. It is evident from the figure that the adhesion of the MWCNTs on sand surfaces is mechanically robust and survives significant agitation. (d) A magnified view of the portion (marked by the white rectangle in (c) of the MWCNT-coated sand particle showing the intact MWCNT growth initiation point on sand surfaces (shown by arrows)

\section{Conclusions}

We have shown that a simple technique of covering sand surfaces using carbon nanotubes can lead to viable materials for capping very soft sediments or sediments contaminated with dense non-aqueous phase liquids, where traditional sand capping is inadequate. Since the sand particles are coated with MWCNTs, their surface properties can also be optimized based on site specific conditions through a mechanistic understanding of the structural and surface chemistry of the material and the sorption/desorption mechanism of various contaminants for this material. The proposed capping material takes full advantage of current developments in nanotechnology yet minimizes the potential ecological risks associated with single nanoparticles. Though this study primarily reports the sorption of chlorinated aliphatics on nano-textured sand, the fact that the sorption capacity of organic compounds on carbon nanotubes is dependent on their molecular structures and follows the general order of aliphatics < nonpolar aromatics $<$ polar aromatics [22], indicates that the carbon nanotube-textured sand can be considerably effective in sorption of other prevalent sediment contaminants in the U.S.A. such as polycyclic aromatic hydrocarbons (PAHs) and polychlorinated biphenyls (PCBs).

\section{Materials and methods}

\subsection{Nano-texturing sand surfaces with multi-walled carbon nanotubes}

We have used an air-assisted vapor phase catalyst delivery chemical vapor deposition (CVD) method to grow MWCNTs on sand particles. In our experiments we used ferrocene (containing iron as the catalyst) and xylene (as the carbon source) for nano-texturing sand surfaces with MWCNTs. A solution of was made by dissolving $1 \mathrm{~g}$ of ferrocene in $100 \mathrm{~mL}$ of xylene. The solution was then vaporized in a steel bottle and was flowed into a furnace using a carrier gas (a mixture of argon/hydrogen (85\% Ar) was used as carrier gas) containing the sand particles to induce MWCNT growth. A variety of parameters (such as growth 
temperature, air flow rate, carrier gas flow rate, etc.) were tested for their effect on MWCNT growth on the sand surfaces.

\subsection{MD simulations}

The molecular dynamics simulations in this paper were performed with the large-scale atomic/molecular massively parallel simulator (LAMMPS) molecular dynamics code [23]. The all-atom assisted model building with energy refinement (AMBER) force field [24] was used for TCE, the extended simple point charge (SPC/E) model [25] was used for water, and the surfaces (MWCNT and hydroxylated silica) were modeled using the optimized potentials for liquid simulations all atom (OPLS-AA) force field [26, 27]. Previous studies have shown the SPC/E water model to be the best available three-point model for determining the interfacial properties of water [28]. In all simulations, the temperature was fixed at $298 \mathrm{~K}$ and controlled with a Nose-Hoover thermostat with a 100-fs damping constant. The SHAKE algorithm was used to constrain the bond lengths of the water molecules. The van der Waals interactions were cut off at $12 \AA$, while the Columbic interactions were calculated using the particle-particle/particle-mesh (PPPM) algorithm.

\subsection{Measurement of sorption coefficients of chlor- inated compounds}

The measurement of sorption coefficients of TCE and other chlorinated compounds on nano-textured sand products were carried out in $42 \mathrm{~mL}$ centrifuge tubes. Approximately $200 \mathrm{mg}$ of nano-textured sand were weighed into each centrifuge tube. The exact mass of the nano-textured sand was recorded. The tubes were then filled to the top with a solution containing $0.5 \mathrm{mmol} / \mathrm{L}$ sodium azide $\left(\mathrm{NaN}_{3}\right)$ and $1.0 \mu \mathrm{mol} / \mathrm{L}$ of the compound under test. Sodium azide was added to inhibit bacterial growth in the tubes. The tubes were closed and then tumbled at room temperature for 7 days to allow the compounds to reach equilibrium between nano-textured sand and the solution. Following the tumbling, the tubes were centrifuged at
$2500 \mathrm{r} / \mathrm{min}$ for $20 \mathrm{~min}$ and $10 \mathrm{~mL}$ of the supernatant was transferred to clean $20 \mathrm{~mL}$ vials and analyzed by gas chromatography to determine the concentrations of the compounds. Sorption coefficients on bare sands were measured similarly, except that approximately $10 \mathrm{~g}$ of sand was used in the measurements. Duplicate or triplicate measurements were carried out. Negative controls (without adsorbents) were also conducted in a similar manner to determine the potential losses of chlorinated compounds such as volatilization during sample transfer or sorption on the tube walls. These unintended losses were taken into account in the calculation of sorption coefficients of chlorinated compounds on bare sand and nano-textured sand. The sorption coefficients of chlorinated compounds on sand or nano-textured sand were calculated as

$$
K_{\mathrm{d}}=\frac{\left(C_{\mathrm{c}}-C_{\mathrm{e}}\right) \times V \times 1000}{m} / C_{\mathrm{e}}
$$

where $K_{\mathrm{d}}$ is the sorption coefficient $(\mathrm{L} / \mathrm{kg}), C_{\mathrm{c}}$ and $C_{e}$ are concentrations of chlorinated compounds in the control and sampling tube, respectively after equilibrium, $V$ is the solution volume $(\sim 41 \mathrm{~mL}$ for nano-textured sand samples and $\sim 36 \mathrm{~mL}$ for sand samples), and $m$ is the mass of adsorbent.

\subsection{Evaluation of critical geotechnical parameters for capping}

One of the essential geotechnical properties of any capping material is their ability to settle down on the sediments on which they are placed. This is a critical issue as far as the viability of ISC materials is concerned. We measured the bulk density $(\rho)$ and porosity $(\varepsilon)$ of nano-textured sand following Standard American Society for testing and materials (ASTM) methods. Briefly, for the measurement of bulk density, a known amount of nano-textured sand was added into a 10-mL graduated cylinder and the volume was read from the cylinder. Bulk density was calculated by dividing the mass by the volume. Porosity was calculated by dividing pore volume by the total volume of the nanotextured sand. Total volumes were read directly from graduated cylinder. Pore volumes were determined 
using the water saturation method. After placing a certain amount of nano-textured sand into a $10-\mathrm{mL}$ graduated cylinder, $5 \mathrm{~mL}$ of water was added to the cylinder and allowed to saturate the sand for at least $3 \mathrm{~h}$. Unsaturated water was then withdrawn with a pipette and the volume of the unsaturated water was determined. Pore volume was calculated as the difference between total water volume $(5 \mathrm{~mL})$ and the unsaturated water volume. The reported values are the average of three replicates. The density of the nano-textured sand $\left(\rho_{\mathrm{ns}}\right)$ was calculated using the equation $\rho_{\mathrm{ns}}=\rho /(1-\varepsilon)$. The calculated density $\rho_{\mathrm{ns}}$ ranged from 1.3 to $1.58 \mathrm{~g} / \mathrm{mL}$. These values of density for the nano-textured sand were significantly less than those measured for the regular sand particles $(2.38 \mathrm{~g} / \mathrm{mL})$ using the same method. The lighter density of the nano-textured sand is consistent with the presence of a highly porous coating of carbon nanotubes on their surfaces. The calculated density values, $\rho_{\text {ns }}$ indicate that the nano-textured sand will settle when placed on the top of the sediment. These results indicate that nano-textured sands possess the geotechnical properties necessary for a capping material.

\subsection{Turbidity test}

To measuring the turbidity, $200 \mathrm{~g}$ of nano-textured sand was mixed with $40 \mathrm{~mL}$ of water in a centrifuge tube and tumbled for $24 \mathrm{~h}$ at $4 \mathrm{r} / \mathrm{min}$. The samples were allowed to settle for $2 \mathrm{~h}$ before the turbidity of the water was measured (using a Hach $2100 \mathrm{~N}$ Turbidimeter) along with its $\mathrm{pH}$.

\section{Acknowledgements}

ST acknowledges Southern Illinois University Carbondale (SIUC) Office of Research and Development Administration for providing financial support through start-up funds and a seed grant.

Open Access: This article is distributed under the terms of the Creative Commons Attribution Noncommercial License which permits any noncommercial use, distribution, and reproduction in any medium, provided the original author(s) and source are credited.

\section{References}

[1] The Incidence and Severity of Sediment Contamination in Surface Water of the United States: National Sediment Quality Survey, $2^{\text {nd }}$ ed.; United States Environmental Protection Agency (EPA): Washington, DC, 2004.

[2] Gustavson, K. E.; Burton, G. A.; Francingues, N. R. Jr.; Reible, D. D.; Vorhees, D. J.; Wolfe, J. R. Evaluating the effectiveness of contaminated-sediment dredging. Environ. Sci. Technol. 2008, 42, 5042-5047.

[3] Sediment Dredging at Superfund Megasites: Assessing the Effectiveness; National Research Council. National Academies Press: Washington, DC, 2007

[4] Zeman, A. J. Subaqueous capping of very soft contaminated sediments. Can. Geotech. J. 1994, 31, 570-577.

[5] Simpson, S. L.; Pryor, I. D.; Mewburn, B. R.; Batley, G. E.; Jolley, D. Considerations for capping metal-contaminated sediments in dynamic estuarine environments. Environ. Sci. Technol. 2002, 36, 3772-3778.

[6] Knox, A. S.; Paller, M. H.; Reible, D. D.; Ma, X.; Petrisor, I. G. Sequestering agents for active caps-remediation of metals and organics. Soil Sediment Contam. 2008, 17, 615-632.

[7] Carbon Nanotubes: Synthesis, Structure, Properties, and Applications; Topics in Applied Physics 80, Dresselhaus, M. S.; Dresselhaus, G.; Avouris, P., Eds.; Springer: New York, 2001.

[8] Endo, M.; Strano, M. S.; Ajayan. P. M. Potential applications of carbon nanotubes. Carbon Nanotubes. Top. Appl. Phys. 2008, 111, 13-61.

[9] Migone, A. D.; Talapatra, S. In Encyclopedia of Nanoscience and Nanotechnology. Nalwa, H. S., Ed.; American Scientific Publishers: Los Angeles, 2004; vol. 4, pp 749-767.

[10] Srivastava, A. A.; Srivastava, O. N.; Talapatra, S.; Vajtai, R.; Ajayan, P. M. Carbon nanotube filters. Nat. Mater. 2004, 3, 610-614.

[11] Upadhyayula V. K.; Deng, S.; Mitchell, M. C.; Smith, G. B. Application of carbon nanotube technology for removal of contaminants in drinking water: A review. Sci. Total Environ. 2009, 408, 1-13.

[12] Li, Y. H.; Zhao, Y. M.; Hu, W. B.; Ahmad, I.; Zhu, Y. Q.; Peng, X. J.; Luan, Z. K. Carbon nanotubes-The promising adsorbent in wastewater treatment. J. Phys: Conf. Ser. 2007, 61, 698-702.

[13] Long, R. Q.; Yang, R. T. Carbon nanotubes as superior sorbent for dioxin removal, J. Am. Chem. Soc. 2001, 123, 2058-2059. 
[14] Rao, G. P.; Lu, C.; Su, F. Sorption of divalent metal ions from aqueous solution by carbon nanotubes: A review. Sep. Purif. Technol. 2007, 58, 224-231.

[15] Chen, C.; Wang, X. Adsorption of Ni(II) from aqueous solution using oxidized multiwall carbon nanotubes. Ind. Eng. Chem. Res. 2006, 45, 9144-9149.

[16] Li, X.; Zhang, X.; Ci, L.; Shah, R.; Wolfe, C.; Kar, S.; Talapatra, S.; Ajayan, P. M. Air-assisted growth of ultra-long carbon nanotube bundles, Nanotechnology 2008, 19, 455609.

[17] Hilding, J. M.; Grulke, E. A. Heat of adsorption of butane on multiwalled carbon nanotubes. J. Phys. Chem. B 2004, 108, 13688-13695.

[18] Mackie, E. B.; Wolfson, R. A.; Arnold, L. M.; Lafdi, K.; Migone, A. D. Adsorption studies of methane films on catalytic carbon nanotubes and on carbon filaments. Langmuir 1997, 13, 7197-7201.

[19] Stan, G.; Bojan, M. J.; Curtarolo, S.; Gatica, S. M.; Cole, M. W. Uptake of gases in bundles of carbon nanotubes. Phys. Rev. B 2000, 62, 2173-2180.

[20] Peterson, E. J.; Huang, Q.; Weber, W. J. Ecological uptake and depuration of carbon nanotubes by Lumbriculus variegatus. Environ. Health Persp. 2008, 116, 496-500.

[21] Zhu, H.; Han, J.; Xiao, J. Q.; Yan, J. Uptake, translocation and accumulation of manufactured iron oxide nanoparticles by pumpkin plants. J. Environ. Monitor. 2008, 10, 713-717.
[22] Chen, W.; Duan, L.; Zhu, D. Adsorption of polar and nonpolar organic chemicals to carbon nanotubes. Environ. Sci. Technol. 2007, 41, 8295-8300.

[23] Plimpton, S. J. Fast parallel algorithms for short-range molecular dynamics. J. Comput. Phys. 1995, 117, 1-19.

[24] Cornell, W. D.; Cieplak, P.; Bayly, C. I.; Metz, K. M. Jr.; Ferguson, D. M.; Spellmeyer, D. C.; Fox, T.; Caldwell, J. W.; Kollman, P. A. A second generation force field for the simulation of proteins, nucleic acids, and organic molecules. J. Am. Chem. Soc. 1995, 117, 5179-5197.

[25] Berendsen, H. J. C.; Grigera, J. R.; Straatsma, T. P. The missing term in effective pair potentials. J. Phys. Chem. 1987, 91, 6269-6271.

[26] Jorgensen, W. L.; Maxwell, D. S.; Tirado-Rives, J. Development and testing of the OPLS all-atom force field on conformational energetics and properties of organic solids. J. Am. Chem. Soc. 1996, 118, 11225-11236.

[27] Jorgensen, W. L. OPLS all-atom parameters for organic molecules, ions, and nucleic acids 5/01. Yale University, New Haven, CT. Personal communication.

[28] Ismail, A. E.; Grest, G. S.; Heine, D. R.; Stevens, M. J.; Tsige, M. Interfacial structure and dynamics of siloxane systems: PDMS/vapor and PDMS/water. Macromolecules, 2009, 42, 3186-3194. 